\title{
Machine Learning Based Learner Modeling for Adaptive Web-Based Learning
}

\author{
Burak Galip Aslan ${ }^{1}$ and Mustafa Murat Inceoglu ${ }^{2}$ \\ ${ }^{1}$ Izmir Institute of Technology, Department of Computer Engineering, \\ 35430 Gulbahce, Urla, Izmir, Turkey \\ bgaslanaieee.org \\ ${ }^{2}$ Ege University, Department of Computer Education and Instructional Technology, \\ 35040 Bornova, Izmir, Turkey \\ mustafa.inceogluaege.edu.tr
}

\begin{abstract}
Especially in the first decade of this century, learner adapted interaction and learner modeling are becoming more important in the area of web-based learning systems. The complicated nature of the problem is a serious challenge with vast amount of data available about the learners. Machine learning approaches have been used effectively in both user modeling, and learner modeling implementations. Recent studies on the challenges and solutions about learner modeling are explained in this paper with the proposal of a learner modeling framework to be used in a web-based learning system. The proposed system adopts a hybrid approach combining three machine learning techniques in three stages.
\end{abstract}

Keywords: adaptive web-based learning, learner modeling, machine learning.

\section{Introduction}

Although the concept of learning from distance have been around for a long time, recent developments in communication technologies and internet itself have opened up a wide variety of possibilities of people who are in need of learning. The concept of web-based learning has been studied for more than a decade and it is closely related with developments in the Internet technology.

Considering the current stage of web-based learning as a preparatory period for the future, most striking advantages of asynchronous distance learning can be emphasized to be used in web-based learning systems [1]. Asynchronous distance learning is based on the fact that the learner should learn at his / her own pace, from any place, and at any desired time. The idea of being able to learn anytime and from anywhere could easily be the most interesting aspects of web-based learning systems. However, the nature of anytime-anywhere learning also opens up new problems such as solicitation of the learner. This handicap can be engaged by deploying proper individualization and personalization mechanisms, which can also be called adaptive web-based learning. 
Adaptivity issue is not only crucial for academic purposes. Popular web-based learning solution providers are also working on improving interaction possibilities for individualization and customization of their service [2]. The adoption of advanced presentation technologies are definitely easier than adopting and / or integrating similarly advanced adaptive mechanisms. Self [3] also remarks on the importance of artificial intelligence techniques in the future of education.

Learner modeling and related issues are explained in the following section of the study. Machine learning for the use of learner modeling is discussed, a survey about proposed solutions on machine learning based learner modeling is given, and a proposal of a learner modeling framework for adaptive web-based learning is given in section 4 .

\section{Learner Modeling in Web-Based Learning}

The generation of learner models resides in the heart of enhancing interaction between the learner and the web-based learning systems. Without the use of proper learner models, web-based learning systems might easily fall into the fallacy of drowning the learner with information flooding in the name of enhancing interaction.

Sison and Shimura [4] define learner modeling as the construction of a qualitative representation called learner model that accounts for learner behavior in terms of a system's background knowledge. Here, learner behavior refers to a learner's observable response to a particular stimulus in a given domain that serves as the primary input to a learner modeling system. The background knowledge consists of the correct facts and principles of the domain, and the misconceptions and errors committed by learners in that domain. The resultant learner model would be an approximate primarily qualitative representation of learner knowledge and skill level in the domain or corresponds to specific aspects of the behavior of the learner.

Webb et al. [5] categorize the purposes for which user models are developed as;

- The cognitive processes that underlie the user's actions,

- The differences between user's skills and expert skills,

- The user's behavioral patterns,

- The user's characteristics.

They also emphasize that first two purposes are usually handled in early applications while user's preferences and behavioral patterns are having been developed since a decade before. The user models that are aimed at exploiting the user's characteristics are rare.

The generation of useful learner models is both a necessity, but also very troublesome to achieve in practice. Aiken and Epstein [6] address this problem by considering that web-based education practices should accommodate diversity and acknowledge that learners might have different learning styles and skill levels. This has been a major goal in many of the education systems that have been developed. Here, they argue that this goal hasn't yet been met because it is a very hard problem. They emphasize on the importance of learning styles with the teachers' point of view, and note that the objective of influencing humans for the better without acknowledging diversity and different learning styles is not possible. Diverse teaching styles are required to stimulate maximum learning and creativity. 
Deploying machine learning techniques over the hard problem of learner modeling also unfolds the concept of intractability. Hence, learner modeling could be considered as a problem that is solvable in theory, but may not be solved in practice for a relatively simple modeling task is clearly intractable. Self [7] considers the case of learner modeling by means of machine learning as a search problem underlying direct machine learning approach to inferring possible cognitive process models.

The debate about learner modeling can be extended to the point of considering the role of the model generated by means of machine learning. Baker [8] describes a learner model as a computational model corresponding to some aspect of the teaching or learning process which is used as a component of an educational artefact. A computational or cognitive model of the learner's problem solving can be integrated into a computer-based learner environment as a learner model. The idea is enabling the system to adapt its tutorial interventions to the learner's knowledge and skills. Such a model-component can be developed on the basis of existing artificial intelligence techniques, and refined by empirical evaluation. The computational model of learner reasoning or problem-solving in a restricted knowledge domain can be used as a component of an intelligent tutoring system that attempts to model the evolution of an individual learner's problem solving processes throughout an interaction between the human learner and the intelligent tutoring system of which the learner model is a component (e.g. the model-component should be able to predict changes in the learner's cognitive states that result from providing some specific knowledge. Baker [8] especially underlines the model-component is precisely a functional component of a tutoring system architecture.

In short, several different studies in literature indicate that learner modeling is the most important yet hardest part of a web-based learning system. The next section of this study approaches this problem from machine learning perspective; pointing out the challenges in using machine learning for learner modeling, and surveying several proposed solutions in literature.

\section{Machine Learning for Learner Modeling}

The main aspect of modeling itself relies on building up a theoretical construct over any kind of process from the real world. Once a model is constructed, reasoning from that model could be made possible with a degree of diverging from real world as several assumptions are held in the generation of that model. Machine learning approaches are widely used for modeling both in industry and academic environments because of the complex relationships that are hard to be represented in mathematical formulation. Considering the variety of information that could be made available when a learner effectively gets involved in a web-based learning system, the modeling of the learner might easily become a quite complicated task. Hence learner modeling by means of machine learning could be an interesting issue for the benefit of adaptive web-based learning systems.

\subsection{Challenges}

Webb et al. [5] also argue that situations in which the user repeatedly performs a task that involves selecting among several predefined options appear ideal for using standard 
machine learning techniques to form a model of the user. The information available to the user can describe the problem and the decision made can serve as the training data for a learning algorithm. The main idea is creation of a model of a user's decision making process that can be used to emulate the user's decisions on future problems.

In spite of such an encouraging point of view for machine learning based learner modeling, it also opens up several serious challenges that should be taken into account. Webb et al. [5] name four main issues of these challenges as: the need for large data sets, the need for labeled data, concept drift, and computational complexity. The learning algorithm does not build a model with acceptable accuracy until it sees a relatively large number of examples, and it imposes a significant importance for the initialization of a proper initial model in the absence of large data sets. The need for labeled data is also an important factor because the supervised machine learning approaches being used require explicitly labeled data, but the correct labels may not be readily apparent from simple observation of the user's behavior. The issue of concept drift takes the potential changes in user's interests and profile into account. As Widmer and Kubat [9] remark in their study, it is important that learning algorithms should be capable of adjusting to these changes quickly. Webb et al. [5] also argues that while academic research in machine learning is often dominated by a competitive race for improved accuracy, computational complexity is a very critical issue for deployment in high-volume real-world scenarios. Computationally expensive algorithms could be interesting if they can be applied in scenarios where models can be learned offline.

\subsection{Proposed Solutions}

There are various implementations of machine learning approaches on learner modeling in literature. The implementations also cover a wide range of educative purposes making use of the data about the learner. The significant studies in literature rely on namely Bayesian networks, neural networks, fuzzy systems, nearest neighbor algorithms, genetic algorithms, etc. and also hybrid systems which consist of combinations of different machine learning techniques.

\section{Bayesian Networks}

Garcia et al. [10] propose a Bayesian network model for detecting learner learning styles based on the learning styles defined by Felder and Silverman [11]. They implemented their study over 27 computer science engineering learners taking an artificial intelligence course. They compared the results of their approach with Index of Learning Styles questionnaire proving that Bayesian networks are effective in predicting the perception styles of the learners with high precision.

Xenos [12] proposes a Bayesian network model to support education administrators and tutors in making decisions under conditions of uncertainty. They implemented their study in one of the modules of an informatics course over approximately 800 learners. The idea is modeling learner behavior in order to make predictions about the success and drop-out rates of the learners for assisting administrators' decisions. They remark on the satisfactory results of the proposed system and proved that it can be a valuable tool in decision-making under conditions of uncertainty. 
Millan et al. [13] propose a Bayesian learner model to be integrated with an adaptive testing algorithm. They tested their study over simulated learners which also had been used in literature before [14], [15], [16]. The results obtained indicate that Bayesian integrated model produces highly accurate estimations of the learners' cognitive states.

Van Lehn and Niu [17] studied the effectiveness of a learner modeler based on Bayesian networks which is used in Andes physics tutoring system [18]. Andes implement a Bayesian solution to the assignment of credit problem by converting the solution graph to a Bayesian Network. The basis of their research is making a sensitivity analysis on the effective performance of the learner modeler in order to understand it better.

Bunt \& Conati [19], [20] studied on the generation of a learner model that can assess the effectiveness of a learner's exploratory behavior in an open learning environment. The learner model is based on Bayesian network, and developed to be a part of The Adaptive Coach for Exploration System [21]. The study is realized with the cooperation of five first-year university learners who have not taken a university math course before. They explain that observations from test results are encouraging.

Reye [22] proposes a learner model structure based on Bayesian belief networks. The idea is gathering information about learners' interactions with the system, and at the same time the model follows the changes in learners' knowledge levels independently of interactions with the system. He remarks on the computational efficiency of a Bayesian belief network based learner model, and points out the advantages both for intelligent tutoring system designers, and for efficient local computation in an implemented system.

Castillo et al. [23] propose an adaptive Bayesian learner modeler rather than a Naïve Bayesian one which has been integrated in the learner modeling module of a web-based learning system named as GIAS. The learner modeling process is based upon Felder and Silverman's Learning Styles [11], and Felder and Solomon's Index of Learning Styles Questionnaire [24]. They compared the adaptive Bayesian learner modeler with the non-adaptive by simulating concept drift [5], [9] scenarios using artificial datasets. Their experimental results proved that implementing an Adaptive Bayesian modeler leads to improvement in dealing with the problem of concept drift.

\section{Neural Networks}

Yeh and Lo [25] demonstrates a neural network based learner modeling approach to be used in computer aided language learning. The learner model processes the learner's browsing behavior over the system using a multi layer feed forward neural network. The number of neurons to be used in the network is settled by means of applying a genetic algorithm for decision. The proposed system is implemented with 46 college freshmen in a freshman English course. The analysis of variance that has been implemented indicates the suitability of the proposed neural network model. It has been addressed that fast execution of neural network makes it possible to assess the learner's meta-cognitive level with real-time immediacy, and it could be used to developed adaptive educational systems.

Villaverde et al. [26] proposes a feed-forward neural network model for exploiting the learning styles of learners. The system aims at classifying the learners based on their learning styles defined by Felder and Silverman [11]. An artificial dataset is 
generated for experimentation by simulating the actions of learners. They emphasize that the information gathering mechanism is imperceptible to learners and the system can recognize learning styles changes over a time period.

Curilem et al. [27] propose a formalization of intelligent tutoring systems, and models the learner preferences with neural networks to be used in an adaptive interface mechanism. The application of the formalization focuses on interface configuration. They emphasize on the importance of implementing didactic ergonomy [28] relevant in the actual context where personalization is considered fundamental for education. Overall system administrates the resources, strategies, and learner models used to build activities.

\section{Other Approaches}

Tsiriga and Virvou [29] propose a framework for the initialization of learner models in web-based educational applications. The main idea is initializing a learner model with the combination of stereotypes, and then the new model of the learner is updated by applying the distance weighted k-nearest neighbor algorithm among the learners that belong to the same stereotype category with the new learner. They implemented the framework on a language learning system called Web-based Passive Tutor [30] with 117 learners belonging to different stereotype categories. The results of the evaluation indicates that with the use of framework, more detailed learner models could be built more quickly as opposed to the non use of such framework.

Andaloro and Bellomonte [31] propose a system called 'Forces' for modeling the knowledge states, and learning skills of the learners in Newtonian Dynamics. The learner data is being recorded, and a fuzzy algorithm is applied to follow the cognitive states the learners go through. The evaluation of the learning process is carried out using an algorithm based on fuzzy set theory.

Huang et al. [32] propose an intelligent learning diagnosis system that supports a web-based thematic learning model. The system processes the log files of the learners and guides the learners in improving their study behaviors as well as helping the instructors on grading with online class participation. While support vector machines, naïve Bayesian, k-nearest neighbor algorithms process the data on learner profile database to update the learner assessment database, the fuzzy expert system works on the learner profile to update both the learner assessment database and learner diagnosis database. The system also predicts the achievement of the learners' final reports. The system is implemented on two fifth grade classes at an elementary school. The experimental results indicate that proposed learning diagnosis system can efficiently help learners on theme-based learning model.

Stathacopoulou et al. [33] propose a neuro-fuzzy learner modeling system to diagnose the errors of high-school learners by collecting the data with simulation tools related to a course, namely vectors in physics and mathematics. The system is tested with simulated learners with different knowledge level categories and their behaviors correspond to fuzzy values. A feed-forward neural network was also trained to for error classification purpose.

\subsection{Future Projections}

Considering that the adaptivity and individualization issues are at utmost importance in today's web-based learning solutions, it will not be surprising that learner modeling 
will be not less, even more important in future studies. Here, McCalla [34] argues that the ability to adapt to users will be a prime feature of any intelligent information system in future, and with vastly enhanced bandwidth, user modeling will be transformed into making sense out of too much knowledge, rather than trying to do with too little. He also argues that since learner modeling activity will be associated with the end application itself, learner models created by end application will exist only as long as the application is active; so many learner models will be created over a span of time as a learner moves from task to task. He also remarks that the need for realistic response times will mean that the ability to reason under resource constraints will be an essential aspect of any model, which is also similar to computational complexity challenge emphasized by Webb et al. [5].

Papatheodorou [35] also remarks that; machine learning offers a suite of powerful techniques either for user model acquisition or for user community induction while supporting complex decision making tasks. He concludes that user modeling should focus on rapid and online learning techniques in future so that small sets of training cases should be evaluated whenever a learner interacts with the system, and the updated user models could be used in upcoming sessions.

\section{Proposed Learner Modeling Framework}

We assume that the structure of web-based learning system should be in multi-layered sense. The multi-layered architecture comprises three layers, namely; the learning management system (LMS), the learner modeling system, and the user interface. As depicted in Fig. 1., the learner modeling system layer is planned to act as a mediator between the user interface and learning management system.

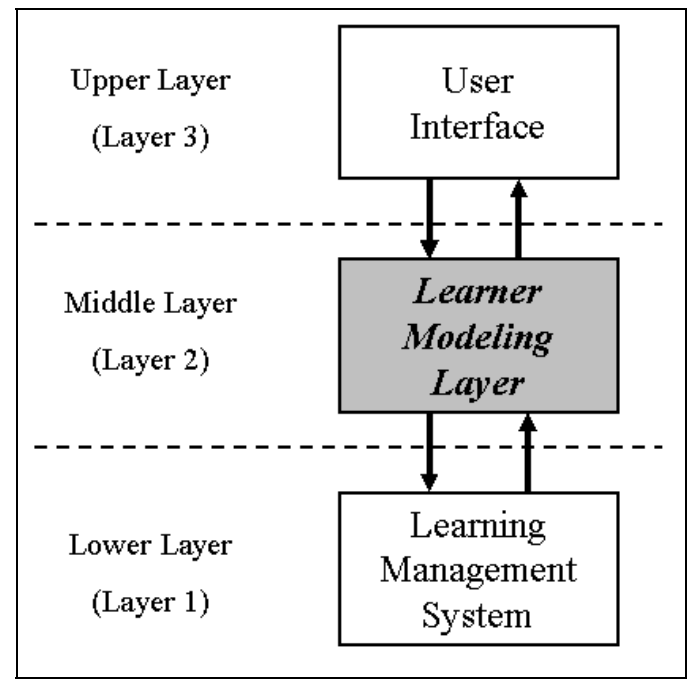

Fig. 1. Layered structure of web-based learning system 


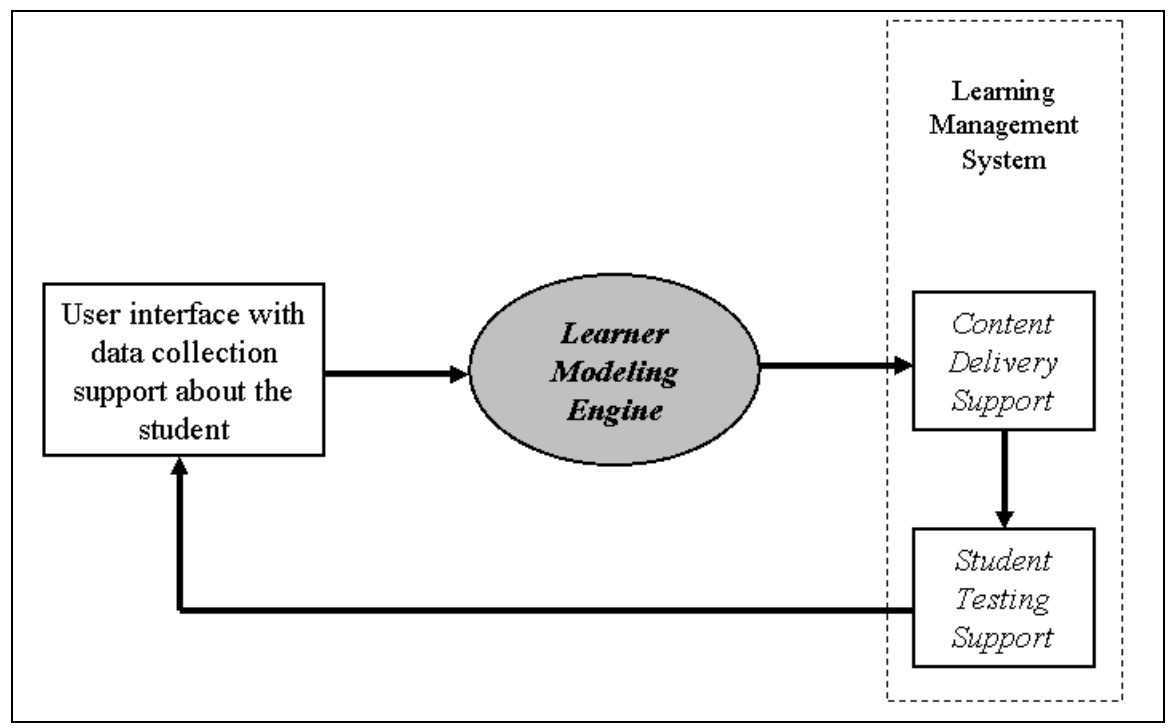

Fig. 2. Another view of web-based learning system proposed in this study

The system works on the basis of processing the incoming learner data from the user interface by the learner modeling system, and triggering the learning management system with the help of learner models generated on the middle layer. Another view of the web-based learning system in layered structure is given in Fig. 2 . The main idea is providing the learner with proper education material to meet his/her learning model.

Designing a learner modeling framework with considerations above can bring up three main questions:

- What should be taught?

- Which learning theory should the learner model be based upon?

- How should the learner model be updated via machine learning?

\subsection{What Should Be Taught?}

Assuming that the below parameters could be important for practicability:

- It should be an interdisciplinary area within the interest of many learners with from very different age groups, so that there will be enough candidates for implementation,

- A wide range of possibility for individualization when compared to other concept areas,

- and the ease of acquiring different teaching materials serving different learning styles.

Considering the above parameters, the teaching of English as a second language (ESL) has been chosen for the first implementation. 


\subsection{Which Learning Theory Should the Learner Model Be Based Upon?}

Felder \& Silverman's learning and teaching styles [11] have been chosen as basis for creating the learner models. This model categorizes the characteristics of learners in 4 sections which are; active/reflective, sensing/intuitive, visual/verbal, and sequential/global. The parameters are not actually binary, e.g. learners are both active and reflective at the same time, but with various tendencies on each side. This model has also been adopted and implemented successfully in several studies [10], [23], [26].

\subsection{How Should the Learner Model Be Updated Via Machine Learning?}

The system proposed consists of three stages. Bayesian networks, fuzzy systems, and artificial neural networks are planned to be implemented as a hybrid system. The overall system architecture is shown in Fig. 3.

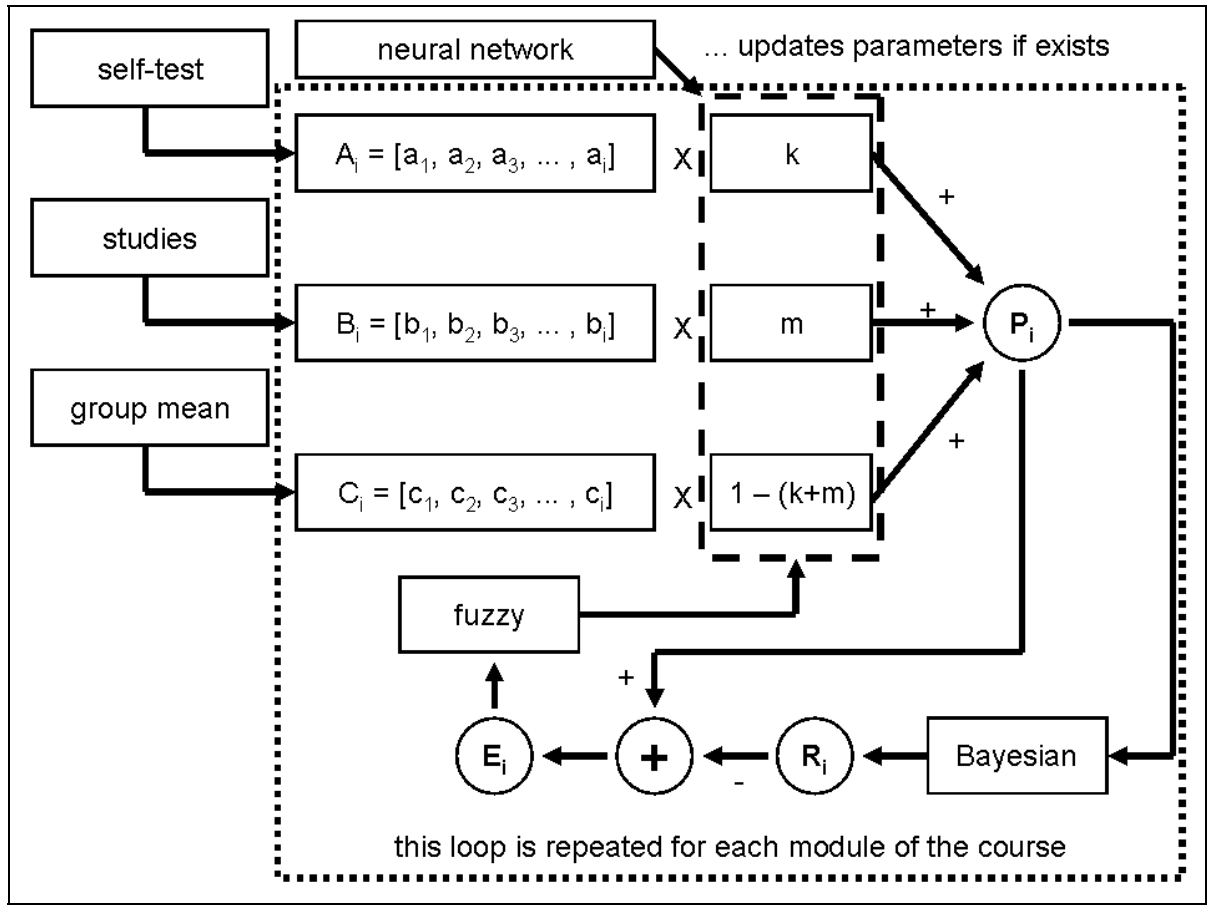

Fig. 3. Overview of learner modeling architecture proposed in this study

\subsubsection{Stage I (Bayesian inference)}

There will be three different parameters to be used in generating a prior belief about the learning style of each user; the self-test, the knowledge from other scientific studies, and the arithmetic mean of the self-test results of the learned group. 
4.3.1.1 Self-test. The learner will be given a self-test (e.g. Index of Learning Styles Questionnaire - ILS [24]) and the results of the test are saved as $A_{i}$ parameter vector of the learner model.

4.3.1.2 The Knowledge from Other Scientific Studies. The learner model will be provided with $B_{i}$ parameter vector which has the learner parameters of similar background and age groups exposed in other studies, such as the study of Montogomery [36] which gives information on the learning styles of junior engineering students.

4.3.1.3 Group Parameters. This is the mean of self-test parameters $\mathrm{A}_{\mathrm{i}}$ vectors of all students who are enrolled for the same course at the same time. This parameter vector, namely $\mathrm{C}_{\mathrm{i}}$, will be saved as another input for the learner modeling system.

- $\mathrm{A}_{\mathrm{i}}$ vector affects with the weight of $\mathrm{k}(0<\mathrm{k}<1)$

- $\mathrm{B}_{\mathrm{i}}$ vector affects with the weight of $\mathrm{m}(0<\mathrm{m}<1)$

- $\mathrm{C}_{\mathrm{i}}$ vector affects with the weight of $[1-(\mathrm{k}+\mathrm{m})]$

and the summation of above three will give the overall prior belief $\left(\mathrm{P}_{\mathrm{i}}\right)$ about the learning style of the learner. The parameters $\mathrm{k}$ and $\mathrm{m}$ can be initialized as $\mathrm{k}=0.33$ and $\mathrm{m}=0.33$ at the startup the learner modeling system.

Assuming that the course consists of modules, (such as 1.1, 1.2, 1.3 etc...) learner behaviors and learner preferences on each module will be recorded and the learning style belief will be updated after the completion of each module as $\mathrm{R}_{\mathrm{i}}$ vector with the help of Bayesian inference. (It was $\mathrm{P}_{\mathrm{i}}=\mathrm{R}_{\mathrm{i}}$ at the startup)

\subsubsection{Stage II (Fuzzy Logic)}

The learner model parameter vector at startup was named as $\mathrm{P}_{\mathrm{i}}$, and the last parameter vector after Bayesian updates was named as $R_{i}$. When the module is completed, the difference between $R_{i}$ and $P_{i}$ vectors will be depicted as an error vector $E_{i}$ and will be fed as input into a fuzzy system with a proper rule base to update the $\mathbf{k}$ and $\mathbf{m}$ parameters depending on the amount of errors in parameter vector. The fuzzy system will update the Pi vector depending on the new $\mathbf{k}$ and $\mathbf{m}$ parameters and this new vector will be the startup learning model parameter vector for the next module.

\subsubsection{Stage III (Neural Network)}

The initial $\mathrm{P}_{\mathrm{i}}$ parameter vector of each learner, and the last updated $\mathrm{P}_{\mathrm{i}}$ parameter vector of the learner after all of the modules of the course has been completed will be recorded in the system. Following the collection of a data set of at least a few course completions, these input-output pairs will be fed into a neural network system as training input. Accordingly when a learner is registered in the system for the first time, his/her first initial Pi vector will be processed via trained neural network the in order to predict the updated version of learning model parameter after the course. In that way, instead of initializing the $\mathbf{k}$ and $\mathbf{m}$ parameters as 0.33 and 0.33 at startup, the predicted neural network weights will be used as initial conditions of $\mathbf{k}$ and $\mathbf{m}$.

However, the stage three is optional, because there has to be enough training data in order to train the neural network. So the third stage is not applicable unless there is sufficient number of completed course data. If there is not enough data for prediction, 
it would be better to apply only the first and the second stages for creating the learner model.

\section{Conclusion}

Individualization and customization issues are becoming more popular in web-based services that have direct interaction with users. Enhancing the computer-learner interaction to increase adaptability of web-based learning systems in the light of proper learner modeling studies is very important for successful implementations of web-based learning systems. It has been proven by the previous studies that adaptivity and adaptability of web-based learning systems positively enhance the interaction with the learners. Considering modeling of the learner as a hard problem, several techniques have been used for different purposes in literature. This paper briefly surveys current trends in machine learning based learner modeling approaches for adaptive web-based learning platforms, and also consists of the challenges and arguments about the future of learner modeling.

In this study, the structure of a web-based learning system is considered in modular sense. The learner modeling framework proposed in this study can have a crucial role as a mediator between the learning management system and user interface. The collected data from the user interface is planned to be processed by learner modeling system for the generation of learner models. In that way, the learner models can trigger the adaptive content organization mechanisms embedded in the learning management system.

While the learner directly interacts with the user interface, the incoming data from the user interface is saved in the system as learner behaviors and learner feedbacks in a given time period. The system is planned to support the modern content delivery standards and to possess intelligent adaptive functions.

\section{References}

1. Inceoglu, M.M., Ugur, A., Aslan, B.G.: Intelligent Approaches for Web-based E-learning Systems. In: Proceedings of the International Conference on Innovations in Learning for the Future, Turkey, pp. 243-250 (2004)

2. Inceoglu, M.M., Ugur, A., Aslan, B.G.: A Review on the Use of Artificial Intelligence in Popular Web-based E-Learning Solutions. In: Proceedings of New Information Technologies in Education Workshop, Turkey, pp. 241-249 (2004)

3. Self, J.A.: Presentation on the Future of Artificial Intelligence in Education, Panel Discussion. In: Eighth International Conference on Artificial Intelligence in Education, Japan (1997)

4. Sison, R., Shimura, M.: Learner Modeling and Machine Learning. International Journal of Artificial Intelligence in Education 9, 128-158 (1998)

5. Webb, G.I., Pazzani, M.J., Billsus, D.: Machine Learning for User Modeling. User Modeling and User-Adapted Interaction 11, 19-29 (2001)

6. Aiken, R.M., Epstein, R.G.: Ethical Guidelines for Artificial Intelligece in Education: Starting a Conversation. International Journal of Artificial Intelligence in Education 11, 163-176 (2000) 
7. Self, J.A.: Bypassing the Intractable Problem of Student Modeling. In: Proceedings of the Intelligent Tutoring Systems Conference. Montreal, pp. 107-123 (1988)

8. Baker, M.: The Roles of Models in Artificial Intelligence and Education Research: A Prospective View. International Journal of Artificial Intelligence in Education 11, 122-143 (2000)

9. Widmer, G., Kubat, M.: Learning in the Presence of Concept Drift and Hidden Contexts. Machine Learning 23, 69-101 (1996)

10. Garcia, P., Amandi, A., Schiaffino, S., Campo, S.: Evaluating Bayesian Networks' Precision for Detecting Student's Learning Styles. Computers \& Education (article in press)

11. Felder, R., Silverman, L.: Learning and Teaching Styles. Journal of Engineering Education 94(1), 674-681 (1988)

12. Xenos, M.: Prediction and Assessment of Student Behaviour in Open and Distance Education in Computers Using Bayesian Networks. Computers \& Education 43, 345-359 (2004)

13. Millan, E., Perez-De-La-Cruz, J.L.: A Bayesian Diagnostic Algorithm for Student Modeling and its Evaluation. User Modeling and User-Adapted Interaction 12, 281-330 (2002)

14. Collins, J.A., Greer, J.E., Huang, S.H.: Adaptive Assesment Using Granularity Hiearchies and Bayesian Nets. In: Lesgold, A., Frasson, C., Gauthier, G. (eds.) ITS 1996. LNCS, vol. 1086, pp. 569-577. Springer, Heidelberg (1996)

15. Van Lehn, K.: Conceptual and Meta Learning During Coached Problem Solving. In: Lesgold, A., Frasson, C., Gauthier, G. (eds.) ITS 1996. LNCS, vol. 1086, pp. 29-47. Springer, Heidelberg (1996)

16. Van Lehn, K., Niu, Z., Siler, S., Gartner, A.S.: Student Modeling from Conventional Test Data: A Bayesian Approach without Priors. In: Goettl, B.P., Halff, H.M., Redfield, C.L., Shute, V.J. (eds.) ITS 1998. LNCS, vol. 1452, pp. 434-443. Springer, Heidelberg (1998)

17. Van Lehn, K., Niu, Z.: Bayesian Student Modeling, User Interfaces and Feedback: A Sensitivity Analysis. International Journal of Artificial Intelligence in Education 12, 154 $184(2001)$

18. Gertner, A.S., Van Lehn, K.: Andes: A Coached Problem Solving Environment for Physics. In: Gauthier, G., VanLehn, K., Frasson, C. (eds.) ITS 2000. LNCS, vol. 1839, pp. 133-142. Springer, Heidelberg (2000)

19. Bunt, A., Conati, C.: Probabilistic Learner Modeling to Improve Exploratory Behaviour. User Modeling and User-Adapted Interaction 13, 269-309 (2003)

20. Bunt, A., Conati, C.: Assessing Effective Exploration in Open Learning Environments Using Bayesian Networks. In: Cerri, S.A., Gouardéres, G., Paraguaçu, F. (eds.) ITS 2002. LNCS, vol. 2363, pp. 698-707. Springer, Heidelberg (2002)

21. Conati, C., Gertner, A., Van Lehn, K.: Using Bayesian Networks to Manage Uncertainty in Student Modeling. User Modeling and User-Adapted Interaction 12, 371-417 (2002)

22. Reye, J.: Student Modelling based on Belief Networks. International Journal of Artificial Intelligence in Education 14, 1-33 (2004)

23. Castillo, G., Gama, J., Breda, A.M.: Adaptive Bayes for a Student Modeling Prediction Task based on Learning Styles. In: Brusilovsky, P., Corbett, A.T., de Rosis, F. (eds.) UM 2003. LNCS, vol. 2702, pp. 328-332. Springer, Heidelberg (2003)

24. Felder, R.M., Soloman, B.A.: Index of Learning Styles Questionnaire (February 2007), http://www.engr.ncsu.edu/learningstyles/ilsweb.html

25. Yeh, S.W., Lo, J.J.: Assessing Metacognitive Knowledge in Web-based CALL: A Neural Network Approach. Computers \& Education 44, 97-113 (2005) 
26. Villaverde, J.E., Godoy, D., Amandi, A.: Learning Styles Recognition in E-Learning Environments with Feed-Forward Neural Networks. Journal of Computer Assisted Learning 22, 197-206 (2006)

27. Curilem, S.G., Barbosa, A.R., De Azevedo, F.M.: Intelligent Tutoring Systems: Formalization as Automata and Interface Design Using Neural Networks. Computers \&Education (article in press)

28. Curilem, G.M.J., De Azevedo, F.M.: Didactic Ergonomy for the Interface of Intelligent Tutoring Systems. Computers \& Education: Toward a Lifelong Learning Society, pp. 7588. Kluwer Academic Publishers, Dordrecht (2003)

29. Tsiriaga, V., Virvou, M.: A Framework for the Initialization of Student Models in Webbased Intelligent Tutoring Systems. User Modeling and User-Adapted Interaction 14, 289 316 (2004)

30. Tsiriga, V., Virvou, M.: Dynamically Initializing the Student Model in a Web-based Language Tutor. In: Proceedings of the First International IEEE Symposium 'Intelligent Systems, vol. 1, pp. 138-143. IEEE Computer Society Press, Los Alamitos (2002)

31. Andaloro, G., Bellamonte, L.: Student Knowledge and Learning Skill Modeling in the Learning Environment 'Forces'. Computers \& Education 30(3/4), 209-217 (1998)

32. Huang, C.J., Liu, M.C., Chu, S.S., Cheng, C.L.: An Intelligent Diagnosis System for Webbased Thematic Learning Platform. Computers \& Education 48, 658-679 (2007)

33. Stathacopoulou, R., Grigoriadou, M., Magoulas, G.D., Mitropoulos, D.: A Neuro-Fuzzy Approach in Student Modeling. In: Brusilovsky, P., Corbett, A.T., de Rosis, F. (eds.) UM 2003. LNCS, vol. 2702, pp. 337-341. Springer, Heidelberg (2003)

34. McCalla, G.: The Fragmentation of Culture, Learning, Teaching and Technology:Implications for the Artificial Intelligence in Education Research Agenda in 2010. Journal of Artificial Intelligence in Education 11, 177-196 (2000)

35. Papatheodorou, C.: Machine Learning in User Modeling. Machine Learning and Applications. In: Paliouras, G., Karkaletsis, V., Spyropoulos, C.D. (eds.) Machine Learning and Its Applications. LNCS (LNAI), vol. 2049, pp. 286-294. Springer, Heidelberg (2001)

36. Motgomery, S.M.: Addressing Diverse Learning Styles Through the Use of Multimedia. In: Frontiers in Education Conference, Session 3a2, pp. 13-21 (1995) 